\title{
A Laboratory Information Management System (LIMS) for an Undergraduate Microchip Fabrication Facility
}

\author{
Paul D. Eckerman and Robert W. Hendricks \\ Virginia Polytechnic Institute and State University
}

\begin{abstract}
We have built an 1,800 $\mathrm{ft}^{2}$ Class 10,000 cleanroom dedicated to teaching the elements of the microchip fabrication process to a multidisciplinary cohort of students from all areas of engineering, science, and even the humanities. This laboratory, equipped with educational tools that allow the fabrication of silicon enhancement mode and depletion mode MOSFETs at about 100 micron rules on 4-inch wafers, is capable of handling up to 500 undergraduates per year-an order of magnitude greater than in typical facilities found at other institutions.

In support of this laboratory, we are developing a laboratory information management system (LIMS) that provides for the paperless operation of the facility. It is designed to track personnel, wafers, supplies, and cleanroom performance and to log wafer processing information and test data. We have based our system on LabWare with interfaces to various types of equipment (e.g., particle counters, semiconductor parameter analysis systems, and barcode readers). Data storage is accomplished with a distributed Microsoft SQL-2000 database residing on a Microsoft Windows 2000 Server; all workstations in the lab operate on Windows 2000 Professional. Access to various views of the data acquired in the laboratory is available to students and laboratory managers via standard World Wide Web browsers. Emphasis has been placed on integration of the acquired data with Microsoft Office 2000, the standard desktop publishing tool required of all students by the University. Data retrieved from the system by students will be processed into basic reports and graphs using Microsoft Word, PowerPoint and Excel. In addition to providing students with information on their individual wafers, the large quantity of data acquired from the hundreds of wafers processed by our students over time provides unique information for incorporation into our accompanying industrial engineering courses in semiconductor manufacturing engineering. Students in these classes will have the opportunity to analyze real-world data from a facility that they have worked in or may easily visit and observe.
\end{abstract}

\section{Introduction}

Virginia Polytechnic Institute and State University, under the auspices of the Virginia Microelectronics Consortium (VMEC), the Bradley Department of Electrical and Computer Engineering, and the Materials Science and Engineering Department, has developed an 1,800 $\mathrm{ft}^{2}$ Class 10,000 cleanroom for teaching the elements of the microchip fabrication process to a multidisciplinary cohort of students from all areas of engineering, science, and even the humanities. ${ }^{1}$ The estimated throughput is approximately 500 students per year (about 170 students per semester). Because of this large number of students, and a resultant large amount of information associated with both materials and supplies as well as processing and test data on our wafers, a system for managing both the day-to-day operations of the facility and a system to track all data collected was required. 
The goal of this system was to provide for a completely paperless system to track wafer processes, tests performed on wafers, cleanroom supplies and materials, and cleanroom performance. Additionally it was desired to create a system that was easily scalable to any cleanroom environment and easy to use and configure for future projects. What follows is a description of the Laboratory Information Management System (LIMS) that was created. The goals of the LIMS were achieved by using such technologies as network-aware testing and monitoring equipment, barcode scanners, and distributed relational databases, all coupled through a TCP/IP intranet, and web reporting technologies to provide access to data from locations outside the lab using a standard World Wide Web (WWW) browser. Because the University has adopted Microsoft Office 2000 as its standard desktop publishing tool for all students and faculty, a system was developed that aided student lab reports by automatically generating tables and graphs of the collected data in formats that are easily imported into MS Word, Excel, and PowerPoint. Tracking of test data and of cleanroom inventory was achieved through LabWare, while particle counts and DI water quality were tracked in Facility Net. All other information was recorded in tables and managed by a Visual Basic application custom created for this system.

\section{System Design}

The design for this system was especially difficult and was critical to the success of the project. An extremely complex problem was targeted, making the design inherently complex.

Additionally, the collection of both wafer test data and cleanroom performance measurements had to be done quickly and with accuracy in order to provide useful reports at a later time.

As has been pointed out by King, ${ }^{2}$ in a commercial wafer fab, the designer must consider such parameters as (and we quote):

1. "Purity and physical conditions of liquids, process gases, and environmental air

2. Safety of personnel, plant, and process equipment

3. Product yield and quantity

4. Community relations with regard to effluent, toxicity, and odors,

5. Governmental agencies requirements for control, measurement, and reporting, and

6. Facility and utility system's condition and operating characteristics security".

He further notes "no single system can perform all of these functions at once". Thus, to assure that the system does not become excessively complex, he recommends several steps that should be considered:

1. The various systems should be as simple as possible and each should take care of a single function.

2. The systems should be microprocessor controlled.

3. Insofar as possible, the same system architecture and components (such as sensors, microprocessors, output signals, etc) should be used.

4. A simple multiplexor function should be used to tie the subsystems together in a central computer system which will deal with alarm, display, recording, trend analysis, preventative maintenance scheduling, mean time before failure analysis, forecasting, etc.

5. Resolution and analysis of data outside the computer system should not be provided. Data should be printed only when it is necessary for mailing or similar purposes. 
Although our laboratory is insignificant when compared to a multi-billion dollar commercial wafer fab, most of the issues raised by King apply in some reduced manner. Furthermore, since it is our goal to provide a small-scale, but realistic, introduction to a wafer fab, we believe that it is essential for our students to have experience with a LIMS system such as the one described here.

The types of users, as well as administrators, of this cleanroom system also affect the level of complexity of the system. The primary users of the LIMS are undergraduate students who have a very limited amount of time to learn how to operate the system before they start entering their data. Therefore, the data entry and retrieval functions must be very straightforward and have a high level of error checking built into them. It is essential to prevent a student from inadvertently entering erroneous data or deleting previously recorded results. The same types of criteria apply to the administrative functions for the LIMS. This system will most likely be administered by advanced undergraduate and graduate students, thus presenting the problem associated with a transient staff. The chances of any given administrator (other than the permanent lab manager) operating the system for more than two years are very slim. Thus, the system must be very simple to operate so that the time required to train new users is minimized. Additionally, the error checking in the LIMS must be extensive throughout and intelligent enough to prevent unintentional changes to either the data or the system configuration while still allowing desired changes to be easily made.

Taking the above constraints into consideration, and following King's recommendations, our system was designed using an approach involving small modules. Each of the modules was built for a specific purpose. By using this approach errors can be isolated to specific areas more quickly, thus making troubleshooting for administrators easier. Additionally, by using commercially available software for some of these modules, a technical support base was already available along with extensive documentation. Since this support base already exists, it aids in learning a new system. Any questions that a new user has can most likely be answered by a technical support document or a helpdesk, rather than having to decipher how programmers solved a problem on a custom built application. A final advantage of implementing the LIMS using small modules is that it provides an opportunity to learn the system piecemeal. One does not need knowledge of the entire system in order to accomplish a single task. Instead, he needs only to know how to use the module specific to the task being performed.

In this implementation, the LIMS was divided into four modules:

1. Wafer Tracking (processing and test results available in laboratory)

2. Wafer Test Reporting (student and lab manager remote access)

3. Inventory Control and Management

4. Cleanroom Performance Monitoring and Alerts

The student users of this LIMS only need to learn two modules - the wafer tracking module and the student wafer test reporting module. The system administrators obviously need to learn all four modules in order to maintain the entire LIMS. The operation of these four modules will be handled by four different applications: LabWare from LabWare, Inc., Facility Net from Particle Measuring Systems Inc., a custom student data entry system using Visual Basic and the LabWare database structure, and a custom web interface for data retrieval. In addition, our wafer testing system, a simplified semiconductor parameter analyzer (SPA), has been coded in LabView from National Instruments. ${ }^{3}$ Our system design accommodates entry of SPA data directly from 
LabView into the SQL-2000 database and retrieval from the database through our custom wafer traveler via the web browser.

LabWare is used for wafer tracking, lab manager wafer reporting, and inventory control. The wafer tracking is assisted by a Visual Basic application running off the LabWare database. This was necessary to incorporate the desired wafer process tracking. Additional tables had to be added to the LabWare database to create a structure able to store a traveler for each wafer that enters the system. This traveler contains all procedures to be performed on the wafer, including etches, oxidations, diffusions, metallizations, and electrical tests, along with results and notes on these processes. It also gives the student a recipe to follow in the creation of a wafer. Traveler details will be discussed further when the specific system implementation is explained.

Facility Net, a program provided by Particle Measuring Systems Inc. for use with their airborne particle counters, handles cleanroom performance monitoring and alarms. Each sensor is accessed via a dedicated Ethernet port and unique IP address. This application will also handle the monitoring of the deionized (DI) water as well as sound alarms if the sensors detect anything out of specifications. DI water is monitored with a Thornton Model 220C system that outputs an RS232C signal that is monitored by Facility Net. A driver for Facility Net was written to listen to the RS232C port for a signal from the DI water sensor and then input the reading to the Facility Net database. By using a custom driver for Facility Net we have centralized all cleanroom performance data tracking operations. This allows us the ability to have a single point of alert for notifying lab managers as well as providing a single source of data regarding any cleanroom failures. When the networked version of Facility Net is used, this information is available on remote computers that have been equipped with the Facility Net client.

In order to complete the paperless tracking system, functionality had to be created for data retrieval by both students and lab managers from outside the cleanroom. This requirement was achieved by utilizing a web-based reporting system. Students are able to access all data collected on each wafer specifically assigned to their lab group for processing using a standard WWW browser. This reporting system has the capabilities of automating the individual applications in the Microsoft Office desktop publishing suite. These capabilities allow for charts and graphs to be automatically created and placed into Office documents that students may later utilize to aid them in the creation of laboratory reports and oral presentations.

\section{System Implementation}

To ease system development, standards were set for the types of personal computers that would be used throughout. The web and database server, a dedicated Dell PowerEdge 300 machine, runs on Microsoft Windows 2000 Server. The server is located outside the cleanroom (and, for that matter, in a different building). The use of Windows allows for the use of Microsoft's Visual Basic for the custom applications, providing a familiar Windows interface for the students and administrators to use, as well as it's ease of code implementation and maintenance. The database for LabWare runs on a Microsoft SQL-2000 server, while the database for Facility Net runs on the Borland Paradox database engine. The WWW server is MS Internet Information Server (IIS) 5.5. All data collection machines in the cleanroom run Microsoft Windows 2000 Professional. Again, with the use of Windows, developers are able to utilize Visual Basic and provide a familiar interface to the system users. Through the use of commercially available software, the 
implementation phase became more of a configuration phase. Facility Net was set up to record data both from the airborne particle counter and the DI water resistivity sensor. The LabWare LIMS was configured to record test data obtained from the testing of resistors, diodes, transistors and sheet resistivity of a wafer directly from the LabView SPA system by utilizing an add-on package provided with LabView. This add-on allows LabView to execute SQL statements and directly insert the acquired data into the LabWare database table structure on the SQL-2000 server.

The most difficult part of the implementation was the creation of the wafer traveler. It was desired to have an electronic traveler that accompanied each wafer, tracing all changes and tests performed on it. Since each mask set used in the lab produces a distinct wafer with differing devices and circuits, it is necessary to perform unique processes and tests based on that mask set. Additional tables had to be added to the LabWare database in order to track not only what tasks had to be performed but to record the results of those processes and tests. In addition to adding tables to track each individual traveler, a table was created to associate each wafer with a group of students for grading purposes. Figure 1 below shows the structure of the additional tables required for the traveler and group tracking records. Further details of our paperless traveler are given elsewhere. ${ }^{4}$

In order to support this additional table structure, custom applications were written in VB to populate the appropriate records and fields with data. When a user logs in, the user ID is

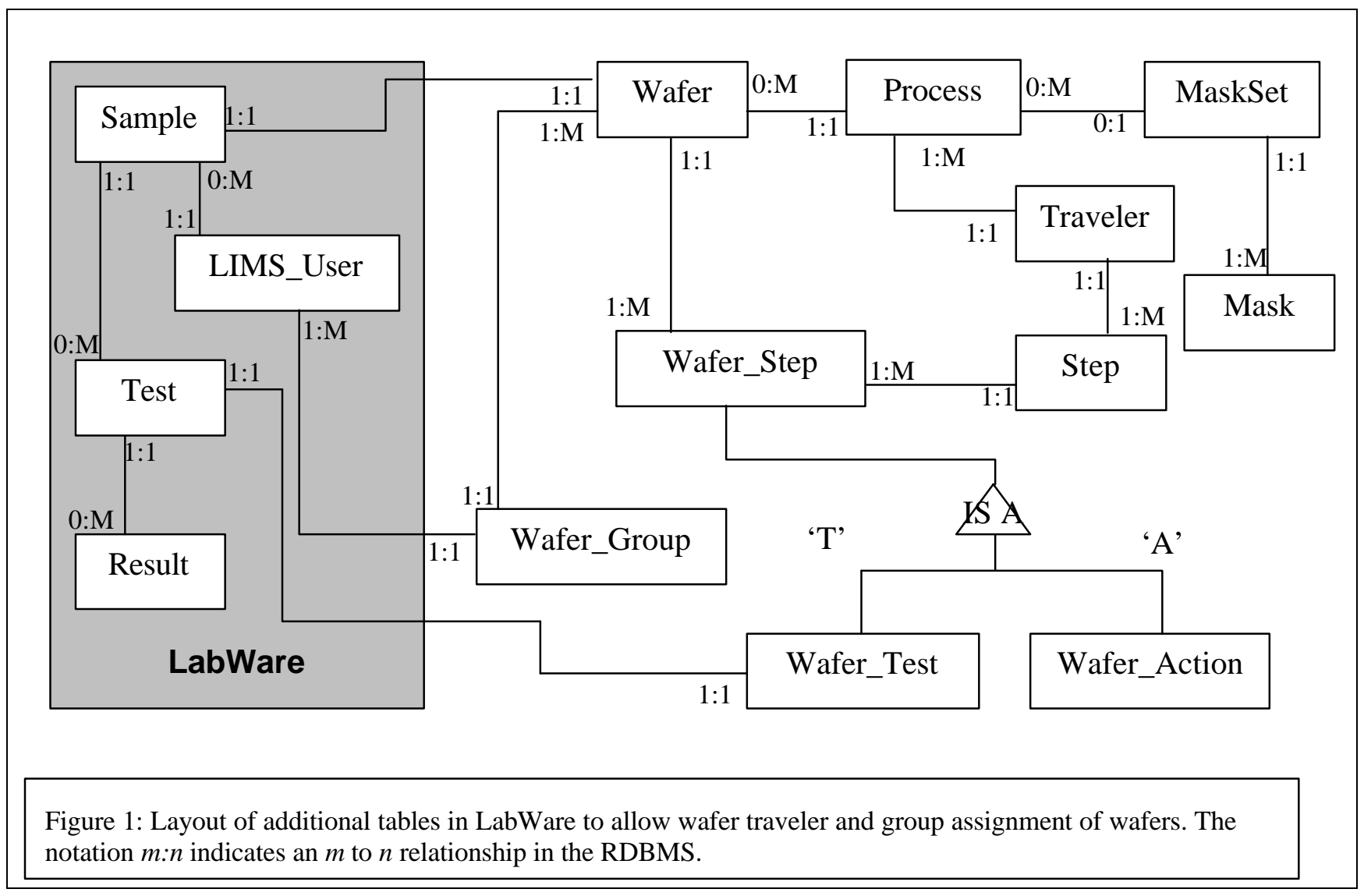


compared to the LabWare 'LIMS_USER' table. This user ID is entered via a barcode reader from the barcode on the student nametag that is worn in the laboratory at all times. If the user ID exists, the user is allowed into the system with the group settings as assigned by the lab manager in LabWare, either as a student, a researcher, or as an administrator. If the user ID does not exist, an error message is generated and the user is not logged into the application. The functions available in the Visual Basic applications vary depending on the group that the user is assigned to. For obvious reasons, it is highly desirable to implement password comparisons in the near future to further limit unauthorized access to administrative functions or to student data.

For members of the student group, only wafer data entry is allowed. Students may only modify database records involving either wafer processes or wafer tests. These modifications are limited to adding new records regarding a wafer or deleting entire wafer records. Modification of test results or other system-generated data is not allowed. Additions to wafer travelers are managed using a bar code system. Each wafer is assigned a unique bar code when it is issued to a student group. In addition, each group is assigned a mask set for their wafer. Each mask in the mask set is assigned a unique mask ID that is also read via a barcode reader. Thus, we are able to check each mask as it is applied to each wafer to assure that all processing steps occur in the proper sequence. Via this process, each wafer is uniquely tied to a specified group of students for each lab session. Thus, when a bar code is read during traveler modification, the system can easily identify which traveler is to be modified. The system is also able to determine what steps in the recipe have already been performed, what the results of those steps were, and what processes remain until to the wafer is considered complete. The use of this bar code system nearly eliminates the issue of user error when entering wafer data, and therefore nearly eliminates the problem of modifying an incorrect traveler.

Members of the research group have all the data entry capabilities of a student plus the rights to modify processes. However, they do not have the rights to make any system-wide changes. Thus, researchers may add new mask sets, set mask revision levels, and add, modify, or delete processing steps in the traveler. Thus, a researcher may develop a new process and test the necessary traveler prior to releasing it for production use.

Wafer test results are obtained from our simplified, but automated, semiconductor parameter analysis (SPA) system that was programmed in LabView. ${ }^{3}$ Wafers are mounted on the appropriate probe station and the desired test is selected on the computer. The tests are performed and the results are sent to the remote LabWare database using standard SQL insert statements available in a LabView add-on. Using this system provides a reliable and very accurate method for recording wafer test data. There can be no user error since all data are handled electronically; the user never has the opportunity to mistakenly enter incorrect data. Furthermore, by using a centralized SQL-2000 RDMS, it is possible to accommodate a number of SPA systems, all of which may save data simultaneously. At the present time, our laboratory has two such test stations. ${ }^{1}$

Students and researchers will use a web-based system to retrieve their traveler from outside of the lab. Since it is University policy that each student own their own state-of-the-art (at the time of purchase!) PC with Windows 98/ME/NT/2K and Office 2000, each has access to their data 
from home and need not rely on a University-provided computer laboratory. Each user ID is bound to one or more wafers so only data from that individual student's wafers can be retrieved via the system's web site. This is done for security purposes; it prevents students from looking at data recorded on a wafer that they have not processed themselves. Access from the web is readonly, thus preventing data entry from anywhere except the systems in the laboratory itself. This procedure minimizes, but does not eliminate, problems associated with "dry-labed" experiments.

Members of the administrator group have all the functionality of the student and research groups as well as access to the administrative functions. These functions include the ability to assign students to groups, add new wafers and assign them to student groups, assign mask sets to groups (one set per wafer), add new student, research, or administrative users, and make current users inactive. For record keeping purposes, no user records are ever deleted from the system. They are simply placed in an inactive user group. To prevent an excess of unused data from taking up valuable space in the system, the system is periodically archived and then unneeded records are purged from the system's database. This archive and purge is accomplished through a standalone application that is triggered by a system-based scheduler. The use of a scheduler eases the responsibilities of the system administrator-they do not have to remember to run the purge utility on any time schedule. Instead, administrators are simply notified of the results once the archive and purge utility has been run.

All other functionality of this LIMS is handled by the various commercial software systems. All inventory tracking is handled by the inventory functions in LabWare. The LabWare interface is also used to add any new tests or to retire old tests to be performed on the wafers. Since LabWare already has the ability to handle these complex functions, the implementation of the system is made simpler. Facility Net is used solely for performance measurements of the cleanroom. The addition of any sensors in the cleanroom requires additional configuration of Facility Net, usually in the form of custom device drivers. Since all sensors are tied to Facility Net, there is a single source for reporting any cleanroom failure, and thus a single source for any alarm or notification triggers. By having a single source for this type of data, any problems can be more quickly resolved because all the necessary data is available at a single point. The ultimate goal of this system is to provide a reporting system that encompasses all of these various commercial systems and is able to create various reports based on data pulled from one or many of the associated databases.

\section{Conclusions}

The Laboratory Management System (LIMS) described in this paper has been designed for the paperless operation of an undergraduate wafer fabrication facility at Virginia Tech. It's primary goal is to provide both students and administrators with an easy to learn, easy to use system that, through automation and web technologies, allows the cleanroom to be run with out the need for notebooks, printouts, or other types of paper administration.

Special care was taken in the design and implementation of this LIMS to ensure that it was both complex enough to manage all desired aspects of the clean room, but also simple enough for students and administrators to learn and become proficient at using in a short period of time. This presented a significant design challenge that was mostly overcome through the use of several commercially available standalone applications. These applications offered a high level 
of functionality, while providing a very good technical support base and a simple user interface. Through some basic configuration, these commercial systems were able to cover most of the requirements set forth for this particular LIMS. The additional functionality desired was met through the use of custom applications, designed to work on top of the existing databases. Thus, an integration of configured commercial software and in-house custom applications was achieved.

In future, as with any software, we anticipate numerous enhancements. The most beneficial enhancements that can be made include improved software security, better integration of functionality between the multiple commercial systems being used, and reporting systems capable of recognizing and analyzing inter-wafer data correlations. Information on yield versus processing parameters will clearly assist us in improving our mask sets and processes to assure that each student group is successful in producing a wafer with working devices-an essential goal if we are to excite students about pursuing careers in microelectronics. Other useful enhancements include automation of cleanroom performance measures. The capability to sound alarms and take preventative measures regarding elevated particle counts and inadequate DI water quality should be automated to the point where lab managers need only receive updates on what is occurring in real time, thus allowing corrective action in a more timely fashion.

\section{Acknowledgements}

We thank LabWare, Inc. (http://www.labware.com) for donating a copy of LabWare and Mr. Clem Padin for his excellent technical support. We thank Particle Measuring Systems (http://www.pmeasuring.com) for substantial discounts on their LasAir II particle counting system and other cleanroom monitoring products and Mr. Ed Applen who wrote the driver for the Facility Net interface to the Thornton DI resistivity meter. Finally, we thank Microsoft (http://www.microsoft.com) for donating an on-going subscription to MSDN that gives us access to all of the Microsoft products used in the project. This project would not have been possible without their generous support.

\section{Bibliography}

1. R. W. Hendricks, An Undergraduate Microchip Fabrication Facility, (this symposium).

2. J. G. King, The Design of Cleanrooms for the Microelectronics Industry, Ch 3 in Cleanroom Design (2E), W. Whyte, ed., John Wiley \& Sons: Chichester, UK (1999), pp. 51-78.

3. D. T. Gray and R. W. Hendricks, A Simplified Semiconductor Parameter Analysis System for an Undergraduate Microchip Fabrication Facility, (2001, to be published).

4. P. D. Eckerman and R. W. Hendricks, A Paperless Wafer Traveler for an Undergraduate Microchip Fabrication Facility, Proceedings $14^{\text {th }}$ Biennial University/Government/Industry Microelectronics (UGIM) Symposium, Richmond, VA, June 18-21, 2001.

\section{PAUL D. ECKERMAN}

Paul Eckerman is an undergraduate Honors student in Management Science and Information Technology at Virginia Tech's Pamplin School of Business and has completed a minor in Computer Science. He gained experience in system design, implementation and maintenance while working at a summer internship with America Online. Paul plans to graduate with a B.S. in May 2001 and continue his work on systems at Infineon Technologies-Richmond as a Computer Integrating Manufacturing Engineer in the 300 mm Diffusion Engineering Group. 


\section{ROBERT W. HENDRICKS}

Robert Hendricks holds a joint appointment as Professor of Electrical and Computer Engineering and Professor of Materials Science and Engineering at Virginia Polytechnic Institute and State University in Blacksburg, Virginia. He is also the Director of the Center for Microelectronics, Optoelectronics, and Nanotechnology (MicrON). Dr.

Hendricks received his B.Met.E (1959) and his Ph.D. (1964) from Cornell University and his M.B.A. (1985) from the University of Tennessee. He joined the faculty at Virginia Tech in 1986 following seventeen years at Oak Ridge National Laboratory and six years with Technology for Energy Corporation. Dr. Hendricks is a Fellow of the AAAS and the APS. He may be reached at robert.hendricks@ vt.edu. 